\title{
Sur le rapport du gouvernement de la Republika Srpska
}

Michèle Picard et Asta Zinbo

\section{(2) OpenEdition \\ 1 Journals}

Édition électronique

URL : http://journals.openedition.org/conflits/2242

DOI : $10.4000 /$ conflits.2242

ISSN : $1777-5345$

Éditeur :

CCLS - Centre d'études sur les conflits lilberté et sécurité, L'Harmattan

Édition imprimée

Date de publication : 1 mai 2007

Pagination : 103-118

ISBN : 978-2-296-03084-8

ISSN : 1157-996X

\section{Référence électronique}

Michèle Picard et Asta Zinbo, "Sur le rapport du gouvernement de la Republika Srpska », Cultures \& Conflits [En ligne], 65 I printemps 2007, mis en ligne le 04 janvier 2010, consulté le 30 mars 2021.

URL : http://journals.openedition.org/conflits/2242 ; DOI : https://doi.org/10.4000/conflits.2242 


\section{Sur le rapport du gouvernement de la Republika Srpska 1}

\section{Michèle PICARD}

Michèle Picard est vice-présidente du Tribunal de grande instance de Paris. Elle a été présidente de la Chambre des droits de l'Homme de novembre 1997 à décembre 2003, après en avoir été membre, de 1996 à 1997. Experte indépendante des Nations unies sur l'Ouzbékistan, elle vient d'être nommée membre $d u$ "Human Rights Advisory Panel in Kosovo » par le représentant spécial du Secrétaire général des Nations unies an Kosovo. A participé à l'ouvrage de Decaux E., Imbert P.H., Petiti L.-E. (dir.), La Convention européenne des droits de l'Homme. Commentaire article par article, Paris, Editions Economica, 1999.

\section{Asta ZINBO}

Asta Maria Zinbo est directrice du Département des initiatives pour la société civile de la Commission internationale pour les personnes portées disparues (ICMP) et travaille sur l'ex-Yougoslavie depuis 1998. De 1997 à 2000, elle était vice-présidente du bureau régional de l'ONG Freedom House basé en Hongrie. Titulaire d'un master en Relations internationales de la Georgetown School of Foreign Service à Washington, un BA (Bachelor of Arts) en science politique de l'université du Michigan, et un diplôme d'études politiques de l'IEP d'Aix-en-Provence.

et article présente les origines et les suites du rapport par lequel le gouvernement de la Republika Srpska (RS) a, en juin 2004, reconnu la réalité du massacre de Srebrenica. A l'origine, suite à une plainte des familles des disparus de Srebrenica auprès de la Chambre des droits de l'Homme de Bosnie-Herzégovine, cette chambre a rendu le 7 mars 2003 la décision «Selimovic» ( ${ }^{\circ} \mathrm{CH} / 01 / 8365$ et al.) sur 49 cas liés au massacre de Srebrenica, qui ordonnait à la RS de faire une enquête sur les événements de

1. Les deux auteures de cet article ne sont engagées que par ce qui concerne la présentation des travaux de leurs institutions respectives, en l'occurrence la Chambre des droits de l'Homme dans la première partie du texte pour Michèle Picard et la Commission internationale pour les personnes portées disparues dans la seconde partie du texte pour Asta Zinbo. 
Srebrenica ${ }^{2}$. Suite à cette décision, le gouvernement de la RS a institué une commission et le directeur général de la Commission internationale pour les personnes disparues (International Commission on Missing Persons ICMP) a participé à ses travaux. En effet, l'ICMP, créée en 1996, pour l'exYougoslavie, a développé des programmes de recherche et d'identification des disparus et de soutien aux associations de familles de disparus, notamment à Srebrenica. La Chambre des droits de l'Homme n'ayant pas été associée aux travaux même de la commission, cet article traitera d'abord du point de vue de la Chambre sur la décision "Selimovic », puis du point de vue de l'ICMP sur la mise en place, le travail et les suites de cette commission.

Quoique fort différentes de nature, la Chambre des droits de l'Homme et l'ICMP ont en commun de conduire leurs actions selon le principe de la défense des droits de l'Homme, c'est-à-dire d'une défense des droits individuels contre les violations d'autorités étatiques. A la fois distinctes et complémentaires des juridictions pénales, la Chambre et l'ICMP considèrent les victimes dans leur individualité et visent la responsabilité collective des gouvernements ou des Etats ou de leurs agents. A la différence des procès et jugements du Tribunal pénal international pour l'ex-Yougoslavie (TPIY), ce sont donc des droits et du sort individuels des victimes de Srebrenica, morts et survivants, et de la responsabilité du gouvernement de la RS que traitent, dans ce texte, la Chambre et l'ICMP.

\section{La décision « Selimovic » de la Chambre des droits de l'Homme}

\section{La Chambre des droits de l'Homme en Bosnie-Herzégovine}

Les accords de Dayton signés en décembre 1995 ont créé une juridiction, la Chambre des droits de l'Homme, chargée d'appliquer notamment la Convention européenne des droits de l'Homme (CEDH) et tous ses protocoles à la Bosnie-Herzégovine. Cette dernière devenait ainsi l'un des pays au monde où, sur le papier, les droits de l'Homme étaient les mieux protégés. Créée au sortir d'une guerre, la Chambre des droits de l'Homme représentait une expérience inédite. Elle était composée de huit juges étrangers désignés par le Conseil de l'Europe et de six juges locaux (deux par nation constitutive) désignés par les deux entités constitutives de la Bosnie-Herzégovine (Fédération croato-bosniaque et RS). Cette Chambre avait compétence sur l'ensemble du territoire et des autorités de Bosnie-Herzégovine. Chaque personne s'estimant victime d'une violation des droits de l'Homme pouvait introduire une plainte auprès de cette Chambre, plainte qui était examinée selon une procédure contradictoire.

2. Human Rights Chamber for Bosnia and Herzegovina, The "Srebrenica Cases" (49 Applications) against THE REPUBLIKA SRPSKA (case $\mathrm{n}^{\circ} \mathrm{CH} / 01 / 8365$ et al.), décision sur l'admissibilité et au fond du 7 mars 2003, accessible sur http://www.hrc.ba/DATABASE/decisions/CH01-8365\%20Selimovic\%20Admissibility\%20and\%20Merits\%20E.pdf 
La première requête que la Chambre a reçue pour des personnes disparues concernait le père Matanovic, prêtre catholique à Prijedor, dans l'ouest de la Bosnie, qui avait disparu avec ses parents en septembre 1995. Il avait été aperçu pour la dernière fois alors qu'il était détenu par la police serbe. Cette requête avait été introduite en son nom et celui de ses parents par l'Ombudsman de Bosnie-Herzégovine ${ }^{3}$. La Chambre a, à cette occasion, défini sa compétence dans le temps. En effet, une convention internationale n'est pas rétroactive et ne s'applique qu'à des événements postérieurs à son entrée en vigueur. La compétence de la Chambre ne pouvait donc s'étendre à des événements antérieurs aux accords de Dayton du 14 décembre 1995. Mais il existe une jurisprudence de la $\mathrm{CEDH}$, qui définit la notion de violation continue des droits de l'Homme, c'est-à-dire une violation ayant pu commencer avant l'entrée en vigueur d'une convention mais se poursuivant après celle-ci. Le père Matanovic et ses parents avaient été aperçus pour la dernière fois en septembre 1995, soit avant l'entrée en vigueur des accords de Dayton, mais leurs noms apparaissaient sur des listes de prisonniers établies après ces accords. La Chambre, qui ignorait alors leur sort, a donc considéré qu'il s'agissait d'un cas de violation continue des droits de l'Homme, et condamné la RS pour violation de l'article 5 de la CEDH en lui ordonnant de mener une enquête ${ }^{4}$.

Par la suite, dans l'affaire Unkovic ${ }^{5}$, des Serbes ont déposé plainte contre la Fédération en raison de la disparition de leur fille et de sa famille. La Chambre a, à cette occasion, redéfini sa compétence en considérant que les souffrances que les gens ressentaient du fait de l'absence d'information sur les membres de leurs familles en faisaient eux-mêmes des victimes. Ce n'était donc plus au nom des disparus que ces personnes introduisaient une plainte, mais en leur nom propre. La Chambre a repris à ce propos la jurisprudence de la CEDH, notamment dans l'affaire "Chypre contre Turquie ». Dans cette affaire, la Cour avait relevé que, vingt ans après les opérations militaires turques dans le nord de l'île, environ 1500 Chypriotes grecs étaient encore portés disparus. Les personnes disparues avaient été vues pour la dernière fois vivantes dans les mains de l'armée turque, mais la Turquie n'avait jamais cherché à savoir ce qu'elles étaient devenues, ce qui constituait donc une violation continue des droits des familles.

L'agent du gouvernement de la Fédération a alors mis la Chambre au défi d'examiner les plaintes des milliers de familles de Srebrenica. C'est ainsi que

3. "Médiateur » international nommé par l'OSCE. Il composait avec la Chambre des droits de l'Homme la Commission des droits de l'Homme prévue à l'annexe 6 des accords de Dayton et avait le pouvoir $\mathrm{d}$ 'introduire des requêtes auprès de celle-ci.

4. Human Rights Chamber for Bosnia and Herzegovina, Josip, Bozana and Tomislav MATANOVIC against REPUBLIKA SRPSKA (case $\mathrm{n}^{\circ} \mathrm{CH} / 96 / 1$ ), décision sur la recevabilité $\mathrm{du}$ 13 janvier 1996, décision au fond du 11 juillet 1997.

5 . Human Rights Chamber for Bosnia and Herzegovina, Djordjo UNKOVIC against THE FEDERATION OF BOSNIA HERZEGOVINA (case n ${ }^{\circ} \mathrm{CH} / 99 / 2150$ ), décision du 12 avril 2002. 
des requêtes sont arrivées à la Chambre par sacs entiers apportés par les associations de Srebrenica. On peut se demander pourquoi une telle masse de requêtes a été déposée devant la Chambre. La raison en est que les victimes n'ont pas de statut devant le TPIY et ne peuvent donc pas se plaindre devant ce tribunal. Ce statut des victimes a d'ailleurs été âprement discuté lors de la création du TPIY et de la Cour pénale internationale. De plus, étant donné l'état déplorable du système judiciaire bosnien après la guerre, il est très difficile de déposer plainte devant les instances nationales. Poussées pour des raisons politiques par les autorités de la Fédération, les familles se sont donc tournées vers la Chambre. Celles-ci étaient d'autant plus désemparées que des rumeurs circulaient encore en Bosnie, selon lesquelles les Bosniaques de Srebrenica seraient prisonniers dans des mines en Serbie ou ailleurs.

\section{La décision «Selimovic» du 7 mars 2003}

Les requêtes sur les cas liés au massacre de Srebrenica ont été introduites de novembre 2001 à mars 2002. En raison de leur nombre, la Chambre, qui avait des moyens limités et ne pouvait les examiner individuellement, a dû faire un tri pour les examiner dans une seule décision, selon une procédure qui avait déjà été suivie à de nombreuses reprises. Les requêtes devaient présenter les mêmes caractéristiques : les victimes devaient être les membres de la famille et non les disparus eux mêmes; les personnes disparues devaient avoir été enregistrées selon les procédures du du Comité international de la Croix-Rouge (CICR) et également déclarées auprès des commissions gouvernementales compétentes; et enfin, selon les juges, les victimes devaient être des civils et non des soldats.

La Chambre a transmis le 20 juin 2002 les 49 requêtes (sur les 1800 introduites) ayant abouties à la première décision dite "Selimovic » au gouvernement de la RS en lui demandant ses observations au regard des articles 3, 8 et 13 de la CEDH, concernant la prohibition des traitements inhumains et dégradants (art. 3), le respect de la vie privée et de la vie familiale (art. 8) et la discrimination (art. 13), et au regard de la Convention sur le génocide de 1948, ratifiée par la Yougoslavie en 1950 et par la République de BosnieHerzégovine le 29 décembre 1992 (et qui figure dans la Constitution de Bosnie-Herzégovine) qui oblige les Etats parties à la Convention à punir les personnes s'étant rendues coupables du crime de génocide.

Les observations transmises par la RS en août 2002 ne portaient que sur la recevabilité et non sur le fond. La RS a notamment fait valoir que les voies de recours internes n'avaient pas été épuisées car les requérants n'avaient pas adressé leurs demandes à la Commission de la RS pour les personnes disparues et que la définition de la déclaration des Nations unies sur les personnes disparues, impliquant que ces personnes devaient avoir été arrêtées, détenues ou kidnappées contre leur volonté, ne s'appliquait pas aux hommes de Srebrenica qui s'étaient volontairement enfui dans les bois. Enfin, la RS a sou- 
tenu que les requêtes étaient incompatibles ratione temporis, les violations étant antérieures aux accords de Dayton.

Dans son examen de la recevabilité des requêtes, la Chambre a fait valoir le fait que, concernant l'épuisement des voies de recours internes, tous les requérants avaient suivi la procédure mise en place par le CICR, selon laquelle toutes les demandes transmises à une partie doivent l'être aux autres. En l'espèce, les requérants avaient formulé des demandes auprès de la Commission d'Etat pour les personnes disparues (en l'occurrence, la Commission fédérale pour les personnes disparues) et parfois du CICR, ce qui était conforme à la procédure précitée. Les voies de recours avaient donc été épuisées. Sur la qualification de personnes disparues, la Chambre a rejeté l'argument de la RS, parce qu'il ressortait clairement du jugement «Krstic »du TPIY que les personnes en question avaient bien été capturées et détenues par les forces serbes avant de disparaître. Sur la compétence dans le temps, la Chambre a fait valoir qu'il s'agissait pour les familles d'une souffrance continue qui n'avait pris fin ni avec les accords de Dayton, ni avec les jugements du TPIY qui ne donnent qu'une description globale des événements. Ces jugements n'empêchent donc pas, au niveau individuel, la persistance des rumeurs et l'attente des familles. De fait, durant la guerre, certains chefs de guerre et certaines milices avaient ouvert leurs propres prisons privées et réduisaient leurs prisonniers à l'esclavage, ce qui alimentait les rumeurs sur le sort des disparus.

Sur le fond, la Chambre a constaté une violation de l'article 8 de la CEDH. En effet, il existe une obligation positive de l'Etat de donner toutes les informations sur le sort d'une personne quand cet Etat possède ces informations ou qu'il les contrôle et qu'il refuse de les donner, arbitrairement et sans justification. Or, il ressortait de la décision « Krstic » que l'armée serbe a séparé les femmes, les personnes âgées et les enfants des hommes en âge de se battre qui, eux, ont été emmenés en détention à Bratunac. L'armée serbe a également capturé des hommes qui tentaient de fuir à travers bois et les a également amenés à Bratunac. Elle a pris leurs cartes d'identité, leurs effets personnels et les a brûlés. Les hommes ont alors été tués lors d'exécutions de masse et enterrés dans des fosses communes. Puis l'armée les a déterrés pour les ré-enterrer ailleurs. Il $\mathrm{y}$ avait donc une action concertée pour cacher les corps. Ces faits, non contestables, démontrent que les autorités de la RS avaient en leur possession ou sous leur contrôle tous les éléments nécessaires pour retrouver, même après la signature des accords de Dayton, des informations sur les personnes disparues. De plus, les autorités de la RS n'avaient pas cherché à trouver ces informations, rien n'avait été entrepris pour aider à la recherche des personnes disparues, aucune enquête n'avait été faite pour déterminer les dates et les lieux de détention, identifier les lieux où les corps avaient été enterrés.

Concernant l'article 3, la Chambre a appliqué la jurisprudence de la $\mathrm{CEDH}$ dans l'affaire «Chypre contre Turquie ». Le niveau de gravité des souffrances 
endurées par les familles de ces personnes était tel qu'on pouvait le qualifier de traitement inhumain, au sens de l'article 3 de la CEDH. La Chambre a appliqué le principe de non-discrimination dès lors qu'une personne est traitée différemment d'autres personnes placées dans une situation identique ou similaire, sans qu'il y ait de justification raisonnable ou objective (qui doit être fournie par l'Etat). En l'espèce, le rapport sur les événements de Srebrenica présenté le 3 septembre 2002 par le Bureau de la RS chargé des relations avec le TPIY était manifestement discriminatoire, en insistant sur le fait que

" on estime qu'à peu près 1800 soldats musulmans ont été tués au combat en fuyant [...] et probablement encore 100 personnes sont mortes d'épuisement physique au cours de leur fuite [...] le nombre de soldats musulmans qui ont été exécutés par les forces Serbes de Bosnie par vengeance personnelle ou par simple ignorance du droit international $[\ldots]$ est probablement inférieur à 1006 ».

Il n'y était fait aucune mention des victimes civiles bosniaques du massacre de juillet $1995^{7}$.

La Chambre a donc constaté une violation des articles 3 (prohibition des traitements inhumains et dégradants) et 8 (respect de la vie privée et de la vie familiale) de la $\mathrm{CEDH}$, et de l'obligation de non-discrimination en relation avec ces dispositions. Elle a ordonné aux autorités de la RS de prendre quatre mesures. La première était de donner toutes les informations en leur possession ou sous leur contrôle sur le sort des personnes disparues et le lieu où elles trouvent, de les relâcher si certaines étaient encore vivantes et détenues, sinon d'indiquer l'emplacement de leur dépouille mortelle. La seconde était de conduire des investigations sur les événements de Srebrenica et de rédiger un rapport sur ces événements. La troisième était de publier la décision de la Cour des droits de l'Homme dans le journal officiel de la Republika Srpska. Enfin, la dernière était de verser à la Fondation du mémorial de Srebrenica-Potocari 8 la première tranche de deux millions de Marks convertibles (KM), sur un montant total de quatre millions de KM (2 $045168 €){ }^{9}$.

6 . «Report about case Srebrenica » (Le rapport sur les événements de Srebrenica), présenté le 3 septembre 2002 par le Bureau de la RS chargé des relations avec le TPIY, pp. 33 et 34, citées au paragraphe 94 de la décision de la Chambre. Voir aussi la déclaration du Dejan Miletic, chef du Bureau de la RS chargé des relations avec le TPIY, lors d'une conférence de presse à Banja Luka, le 3 septembre 2002.

7. Ce rapport a été fortement condamné par la communauté internationale, les responsables politiques, et les ONG (voir Oslobodjenje, 4 septembre 2002, pp. 4-5 ; Dnevni Avaz, 4 septembre 2002, p. 4). Le bureau du Procureur du TPIY a exprimé son amertume envers « ce rapport scandaleux et honteux, dans lequel l'ensemble des preuves bien établies présentées devant le TPIY a été totalement ignoré ", (déclaration de Graham Blewitt, dans Dnevni Avaz, 5 septembre 2002, p. 9, Jutarnje Novine, 5 septembre 2002, p. 6).

8. Fondation créée par décision du Haut Représentant le 10 mai 2001 (voir le communiqué de presse du bureau du Haut Représentant (OHR) du 10 mai 2001, accessible sur http://www.ohr.int/).

9. Voir Human Rights Chamber for Bosnia and Herzegovina, The "Srebrenica Cases" (49 Applications) against THE REPUBLIKA SRPSKA, op.cit., IX. Conclusions, article 220.10, 
Il est possible d'expliquer le sens de cette décision sans violer le secret des délibérations. Les juges de la Chambre ont beaucoup réfléchi à ce que pouvait être une réparation face à une tragédie aussi épouvantable et à l'impossibilité de jamais satisfaire les victimes. Ils ont d'abord pensé à donner l'argent directement aux associations qui représentent et aident les victimes de Srebrenica. Mais de tels conflits opposent ces associations entre elles qu'il est impossible de désigner un seul représentant légitime des victimes. Concernant l'obligation de mener une enquête sur les événements, les juges n'avaient pas mentionné la création d'une commission mais avaient à l'esprit la Commission vérité et réconciliation d'Afrique du Sud. Il manquait en effet en Bosnie une commission publique et non criminelle, qui n'établisse pas des responsabilités pénales, afin de faciliter une véritable enquête, non polémique. Suite à la suggestion de la Chambre, la RS a proposé de créer une commission. Lors de l'examen du suivi de la décision, la Chambre a insisté auprès du Haut Représentant sur la nécessaire transparence des travaux de la commission et notamment sur la tenue d'auditions publiques, ce qui n'a pas été le cas dans la réalité. Il y a lieu de rappeler qu'il existait déjà un Tribunal pénal international et que les juridictions locales étaient également compétentes pour juger des crimes de guerre. Il était non seulement inutile de redoubler leurs travaux, mais la commission souhaitée par la Chambre en différait aussi grandement dans son principe. Un tribunal pénal examine des responsabilités pénales individuelles et, notamment dans le cas du TPIY pour Srebrenica, n'envisage le sort des victimes que globalement. Par contraste, et conformément au principe de la défense des droits de l'Homme, les juges de la Chambre voulaient qu'il y ait une véritable enquête et une reconnaissance de responsabilité collective de la part du gouvernement de la RS. Il s'agissait de déterminer en quoi le gouvernement de la RS, et non tel ou tel individu, était responsable. Au demeurant, il était facile pour la RS de se réfugier derrière la responsabilité individuelle de tel ou tel soldat et de l'auditionner sur les événements, ce qui n'aurait fait que retarder indéfiniment tous les travaux de la commission.

\section{Les suites de la décision de la Chambre des droits de l'Homme}

La décision a été diversement accueillie. Les réactions de la communauté internationale, des ONG de défense des droits de l'Homme et de la presse internationale anglo-saxonne ont été positives, la presse française s'en étant complètement désintéressée. C'est l'association des mères de Srebrenica et de la Podrinje, dirigée par Ibran Mustafic et opposée aux accords de Dayton, qui a émis les critiques les plus violentes ${ }^{10}$. Elle a reproché à la Chambre d'être passée à côté de la question, puisque les vraies victimes n'étaient pas les proches, mais les disparus, et que la violation des droits de l'Homme était le mas-

\footnotetext{
demandant à la RS de verser $2000000 \mathrm{KM}$ au plus tard le 7 septembre 2003 et quatre paiements annuels de $500000 \mathrm{KM}$ à payer le 7 septembre 2004, 2005, 2006 et 2007.

10. Jutarnje novine, 10 mars 2003.
} 
sacre lui-même et non le fait que la RS n'ait pas dit où se trouvaient les corps. De plus, elle était opposée au fait de recevoir de l'argent de la RS pour le cimetière et le mémorial de Potocari. De telles réactions sont humainement tout à fait compréhensibles, même si les bases du raisonnement juridiques sont différentes. Les quotidiens de la Fédération Dnevni Avaz et Oslobodjenje ont repris les mêmes arguments, et l'hebdomadaire Dani a demandé s'il était bien naturel de réclamer aux criminels d'enquêter sur leurs propres crimes. Ces journaux ont cependant approuvé le fait que les contribuables serbes aient à payer pour les victimes bosniaques, alors que beaucoup des femmes de Srebrenica ont considéré qu'il aurait été préférable que la compensation soit payée directement aux victimes, individuellement et non collectivement.

Concernant l'exécution de la décision, la Chambre n'avait aucun pouvoir coercitif, d'autant qu'elle a pris cette décision en mars 2003 et a cessé d'exister fin 2003, mais elle a néanmoins coopéré avec le Haut Représentant à ce sujet. Les premiers versements financiers de la RS pour un montant de deux millions de KM ont été faits le 4 septembre 2003. La décision a été publiée dans le journal officiel de la RS. Ses représentants ont présenté des excuses publiques en mai 2004. Suite à la décision de la Chambre, le gouvernement de la RS a présenté deux rapports. Le premier rapport intérimaire, publié le 6 juin 2003. La RS a publié un deuxième rapport intérimaire le 5 septembre 2003, qui n'apportait rien de nouveau et qui insistait essentiellement sur l'aspect pénal, ce qui ne correspondait pas à la demande de la Chambre ${ }^{11}$.

\section{La commission d'enquête de la RS et le rapport de la RS sur les événe- ments de Srebrenica : le point de vue de l'ICMP}

\section{Le travail de la commission}

La Chambre ayant considéré que ces deux rapports intérimaires n'avaient pas rempli les obligations de la RS, le Haut Représentant en BosnieHerzégovine, Paddy Ashdown, a annoncé pendant une conférence de presse du 15 octobre 2003 que le président de la RS, Dragan Cavic, devait former une commission chargée de rendre un rapport complet dans les six mois. Les victimes et d'autres membres de la communauté bosniaque ont exprimé leur grande inquiétude et leur crainte que cette commission soit de mauvaise foi et que leur participation puisse donner une légitimité indésirable au processus ${ }^{12}$. En décembre

11. Il est à noter que suivant le principe de l'universalité des droits de l'Homme, la Chambre a ultérieurement pris d'autres décisions concernant d'autres régions de Bosnie-Herzégovine et impliquant également la responsabilité de la Fédération. Pour toutes les décisions de la Chambre des droits de l'Homme, voir : http://www.hrc.ba/

12. Plusieurs survivants bien connus, comme Hasan Nuhanovic, ou d'autres figures publiques, comme Amor Masovic, président de la Commission fédérale pour les personnes disparues, ont été proposés mais ils n'ont pas accepté de participer à la commission (entretien de Asta Zinbo avec Hasan Nuhanovic, le 20 novembre 2003). 
2003, après de longues discussions sur la composition de cette commission, Marko Arsovic, Milan Bogdanic, Milorad Ivosevic, Djordje Stojakovic et Gojko Vukotic ont finalement été nommés comme représentants de la RS, Smail Cekic, directeur de l'Institut pour la recherche sur les crimes contre l'humanité et le droit international, comme représentant de la communauté des victimes, et Gordon Bacon, directeur général de la Commission internationale pour les personnes portées disparues (ICMP), comme représentant de la communauté internationale. M. Bacon était membre de la commission à titre personnel. Le 25 décembre 2003, le gouvernement de la RS a confirmé la nomination des membres de cette commission, dont l'intitulé exact était «Commission pour l'enquête sur les événements à Srebrenica et dans ses environs entre le 10 et le 19 juillet 1995 ».

Au début de l'année 2004, après certains retards dans sa mise en place, la commission a déterminé ses règles de procédure (dont une procédure de prise de décision par consensus), sa stratégie et son plan de travail. Les membres de la commission ont adressé des demandes à diverses institutions telles que l'armée et la police, et ont essayé de recevoir des archives, mais se sont parfois retrouvés avec des dossiers quasiment vides, personne ne semblant avoir eu en sa possession les documents pertinents. Ils ont alors pris conscience de l'influence clandestine de certains officiels de la RS. Plusieurs membres de la commission ainsi que leurs familles ont été menacés, se sont fait cracher dessus ou ont été qualifiés de traîtres au peuple serbe et à leur patrie. La combinaison des manipulations politiques, de l'hostilité de l'opinion publique, du manque de moyens et de soutien officiel et des menaces contre la sécurité des membres de la commission représentait une obstruction à son travail ${ }^{13}$.

Suite aux inquiétudes exprimées par le Haut Représentant, le gouvernement de la RS lui-même et le rapport intérimaire du 15 avril 2004, le Haut Représentant a annoncé que le gouvernement de la RS devait destituer M. Arsovic, le président en exercice de la commission, et que celle-ci devait recevoir un soutien convenable dans son travail. Le Haut Représentant a en outre annoncé la destitution de Dejan Miletic, officier de liaison de la RS avec le TPIY, et du général Cvjetko Savic, chef d'état-major de l'armée de la RS, en raison de leur obstruction aux travaux de la commission ${ }^{14}$. Enfin, le Haut Représentant a publiquement réprimandé la commission et a rendu le président de la RS, Dragan Cavic, et le Premier ministre de l'époque de la RS, Dragan Mikerevic, personnellement responsables du succès de ses travaux. Ces événements ont fait l'objet d'une importante couverture médiatique.

La commission a reçu l'instruction du Haut Représentant d'éviter les développements historiques sur les événements antérieurs au massacre et de se

13. Lettres de la commission au gouvernement de la RS datées du 19 mars et du 8 avril 2004.

14. Décisions du Haut Représentant n²03/04 et 204/04 du 16 avril 2004, accessibles sur http://www.ohr.int/ 
concentrer sur la recherche de nouvelles informations, notamment à propos des localisations possibles de nouvelles fosses communes et de la liste des personnes exécutées entre le 10 et le 19 juillet $1995^{15}$. La commission a alors révisé sa stratégie et son plan de travail. Des équipes de deux rapporteurs ont été formées : M. Bogdanic et Bacon ont été chargés de rechercher l'emplacement possible des fosses, M. Cekic et Stojakovic d'obtenir de la documentation du ministère de l'Intérieur et de la police ; M. Ivosevic et Vukotic d'enquêter sur le rôle de l'armée, ou plutôt des armées. A la fin du mois de mai, le gouvernement de la RS a nommé un septième membre, Zeljko Vujadinovic.

Lors de la préparation du rapport final, la commission a reçu un grand nombre de documents de la police et de l'armée de la RS. Certains de ses membres, en coordination avec les commissions gouvernementales de la Fédération et de la RS pour les personnes disparues, sont allés vérifier sur le terrain l'emplacement supposé des fosses. Le rapport final de la commission a été soumis le 4 juin 2004 au gouvernement de la RS, qui l'a adopté le 11 juin 2004 16. Il contenait des informations sur la localisation de 32 fosses communes, dont huit jusqu'alors inconnues et trois sans données assez précises pour pouvoir les localiser, ainsi que des informations sur le statut d'environ 1849 cas examinés par la Chambre des droits de l'Homme, dont 1194 personnes considérées comme ayant disparu entre le 10 et le 19 juillet 1995. Le rapport développait en détail le rôle des unités de l'armée et de la police du ministère de l'Intérieur de la RS, en incluant les documents qui rendaient claires les trois phases de l'opération «Krivaja » : l'attaque, la séparation des femmes et des enfants, l'exécution des hommes. Il comprenait également les ordres pour la participation d'unités de police de la République serbe de Krajina (RSK) et de la Serbie ${ }^{17}$. La documentation reçue, dont une partie était contenue dans les annexes confidentielles, provenait généralement des institutions de la RS ainsi que de certaines institutions fédérales et internationales. Les données recueillies durant le travail de la commission, en sus des listes de personnes disparues, consistaient en documents provenant de structures de l'armée et de la police, telles que le ministère de la Défense et le ministère de l'Intérieur. Il s'agissait d'ordres de mouvements pour des unités militaires, d'ordres à des compagnies pour qu'elles fournissent des véhicules, des bus et de l'équipement à certaines dates avec indication que le but en était l'opération « Krivaja 95 », d'inter-

15. Intervention de Milan Bogdanic, président de la commission, pendant la conférence du ICMP «Mécanismes pour la recherche et la documentation de la vérité » (« Mechanisms for Seeking and Documentation of the Truth»), organisée le 20 octobre 2004 à Jahorina.

16. Voir Republika Srpska Government - The Commission for Investigation of the Events in and around Srebrenica between $10^{\text {th }}$ and $19^{\text {th }}$ July 1995, The Events in and around Srebrenica between $10^{\text {th }}$ and 19th July 1995, Banja Luka, 11 juin 2004, accessible sur: http://www.vladars.net/pdf/srebrenicajun2004engl.pdf

17. Lettre du Haut Représentant Paddy Ashdown à Javier Solana (Haut Représentant pour la Politique étrangère et de sécurité commune de l'UE) et Jaap de Hoop Scheffer (secrétaire général de l'OTAN), résumée dans le communiqué de presse du OHR du 11 juin 2004, accessible sur http://www.ohr.int/ 
views ou de déclarations de témoins, tels que des chauffeurs de bus ou de camions décrivant le nombre de personnes qu'ils avaient transportés, dans quel lieu, suite à quoi il avaient entendu des coups de feu, puis plus rien. Mais toutes les informations demandées n'avaient pas été reçues et, le 16 juin 2004 seulement, le ministère de l'Intérieur de la RS a fourni seize boîtes de documents. Un délai a donc été accordé jusqu'au 15 octobre 2004 pour la préparation d'un addendum au rapport ${ }^{18}$.

Face à la documentation détaillée qu’ils avaient trouvée, les membres de la commission ont eu, aux dires de l'un des participants, des réactions de choc, de surprise et de peur. Ils ont officieusement trouvé des preuves concernant 8200 personnes portées disparues suite à la chute de Srebrenica. Les avocats, les juges et les historiens renommés ayant participé au travail de la commission avaient déjà reçu des menaces et se demandaient comment il leur serait possible de publier ces informations tout en continuant à vivre à Banja Luka ${ }^{19}$.

L'addendum au rapport final a été complété et soumis le 15 octobre 2004. L'essentiel de cet addendum est constitué par une tentative d'établir une liste complète des personnes portées disparues. La commission a créé une base de données spéciale et y a enregistré des milliers d'entrées provenant de multiples listes déjà existantes. Les difficultés rencontrées provenaient de la similitude entre certains noms propres, du caractère incomplet ou erroné de certaines données, obstacles déjà rencontrés lors de tentatives précédentes pour établir de telles listes par recoupement. Les listes ont été réparties en plusieurs souscatégories, mais le rapport indiquait que les données variaient entre 7000 et 8000 personnes pour la période de juillet $1995^{20}$, et que 13569 personnes sont représentées sur les listes couvrant la période de 1992 à 1995 dans la région de Srebrenica ${ }^{21}$. Dans tous les cas, selon diverses sources, les données ont intentionnellement été morcelées, par peur des réactions de l'opinion publique et de conséquences fâcheuses pour les membres de la commission.

18. Décision du gouvernement de la RS, lors de sa $87^{\mathrm{e}}$ session le 16 juillet 2004 , de prolonger le travail de la commission.

19. Commentaire d'un membre de l'équipe locale qui a assisté au travail interne de la commission en tant qu'assistante de Gordon Bacon.

20. Voir Republika Srpska Government - The Commission for Investigation of the Events in and around Srebrenica between $10^{\text {th }}$ and $19^{\text {th }}$ July 1995, Addendum to the Report of the $11^{\text {th }}$ June 2004 on the Events in and around Srebrenica between 10th and 19th July 1995, Banja Luka, 15 octobre 2004, p. 9, accessible sur http://www.vladars.net/pdf/srebr_final_e.pdf. La liste A1 comporte les noms de 7108 personnes disparues entre le 10 et le19 juillet 1995, la liste A2 ceux de 698 personnes disparues en juillet 1995 à cause des événements survenus entre le 10 et le 19 juillet 1995, la liste B ceux de 936 personnes pour laquelle les informations sur la date de disparition (ayant eu lieu ou non entre le 10-19 juillet 1995) sont contradictoires, la liste C1 ceux de 4556 personnes pour laquelle la date de disparition est en dehors du mandat de la commission, et la liste C2 ceux de 271 personnes qui ont été enregistrées comme disparus et qui ensuite se sont révélées vivantes ou bien être mortes dans des circonstances ne relevant pas du mandat de la commission.

21. Addendum to the Report of the 11th June 2004..., op.cit., p. 8. 
La commission s'est donc focalisée sur les 1149 demandes adressées à la Chambre des droits de l'Homme dans un cadre temporel précis et dans ses conclusions a également recommandé qu'une analyse plus approfondie soit faite par des institutions appropriées.

Lors de sa session du 28 octobre 2004, le gouvernement de la RS a accepté le rapport qui "indique sans aucun doute possible que des crimes de grande envergure ont été commis dans la région de Srebrenica en juillet 1995, en violation grave du droit humanitaire international 22 ». Il a également déclaré qu'il compatissait avec la douleur de tous les proches des victimes de Srebrenica, et a exprimé ses condoléances sincères et ses excuses pour la tragédie qu'ils avaient vécue. Il a affirmé son engagement à traduire en justice toutes les personnes ayant commis des crimes de guerre et déclaré que la méthode de travail de la commission devrait servir d'exemple pour des enquêtes ultérieures sur des crimes commis dans d'autres régions, quels qu'en soient les auteurs.

\section{Le suivi du rapport et les perspectives politiques en Bosnie-Herzégovine}

Suite à la demande du Haut Représentant adressée en janvier 2005 au Premier ministre de la RS, un groupe de travail de huit personnes a été nommé pour mettre en œuvre les conclusions du rapport final de la commission sur le massacre de Srebrenica. Il devait en analyser la documentation et identifier tous les officiels dont les noms apparaissaient dans les annexes confidentielles, notamment ceux qui étaient toujours employés dans les institutions de la RS. Dans son rapport du 1er avril 2005, le groupe de travail a identifié 892 personnes toujours employées comme fonctionnaires au niveau des services des municipalités, des entités ou de l'Etat central, surtout dans les services du ministère de l'Intérieur et de la police, en dépit de leur implication dans les événements de Srebrenica. Ce rapport a été transmis au bureau du procureur de Bosnie-Herzégovine et au bureau du Haut Représentant, les noms y figurant ne devant pas être rendus publics tant qu'une procédure officielle n'aurait pas été ouverte. Les membres des associations des familles des disparus de Srebrenica, entre autres, ont fait appel à plusieurs reprises pour que cette liste des personnes impliquées soit rendue publique. La polémique a continué jusqu'à la publication de 69 noms dans la presse le 24 août 2006 et de 59 noms supplémentaires le lendemain, sur un total de 810 personnes, en dépit des déclarations du bureau du procureur de la Cour de Bosnie-Herzégovine s'opposant à cette publication, en raison de l'enquête en cours sur les crimes de guerre à Srebrenica et du droit des accusés à se défendre ${ }^{23}$. Le bureau du procureur du TPIY a dû ajouter aussi des clarifications publiques concernant le

22. Government of the Republic of Srpska, Conclusions, Banja Luka, 28 octobre 2004, conclusion $\mathrm{n}^{\circ} 2$, document accessible sur http://www.vladars.net/pdf/srbren_zaklj_e.pdf

23. Oslobodjenje, 24 août 2006, pp. 4-5, et 25 août 2006, p. 7. 
fait que le rapport de la commission devrait être rendu public, mais surtout pas les parties confidentielles, contenant par exemple les noms des personnes impliquées, sans l'accord des institutions judiciaires ${ }^{24}$.

Le 6 avril 2005, le nouveau Premier ministre de la RS, Pero Bukejlovic, a déclaré que l'adoption de ce rapport montrait la détermination du gouvernement de la RS à faire toute la lumière sur les événements de Srebrenica. Le Haut Représentant a reconnu le sérieux du travail accompli, mais a regretté qu'il ne soit pas complet. En effet, le ministère de l'Intérieur de la RS n'a pas fourni d'information sur des centaines de personnes déployées en juillet 1995 à Srebrenica sous le contrôle de l'armée de la RS.

Parallèlement, en mars 2005, Pero Bukejlovic déclarait que le génocide commis contre les Serbes à Sarajevo était peut-être plus grand qu'à Srebrenica ${ }^{25}$, ce qui a été considéré par certains comme une provocation politique visant à détourner l'attention de l'échec des autorités de la RS à extrader Karadzic et Mladic. Les représentants de plusieurs ONG ont fortement condamné cette déclaration 26. Selon Sulejman Tihic, membre de la présidence de Bosnie-Herzégovine, de tels propos prouvaient la nécessité d'établir une Commission vérité et réconciliation pour la Bosnie, qui examinerait les souffrances de tous les civils ${ }^{27}$. La Chambre des représentants du Parlement de la Fédération a quant à elle adopté une motion condamnant ces propos comme étant «des mensonges horribles 28 ».

Après l'apparition dans les rues de Banja Luka d'affiches niant les crimes commis à Srebrenica par l'armée de la RS, le président de la RS Dragan Cavic a déclaré le 9 juin 2005 qu' «il n'est pas fondé de parler de génocide 29 » à propos de Srebrenica. En dépit des rapports soumis par la commission de la RS, du jugement Krstic du TPIY ayant conduit à une condamnation pour génocide, et de la divulgation en juin 2005 de bandes vidéo d'exécutions par l'unité paramilitaire des «Scorpions », les officiels de la RS ont continué à minimiser la gravité des crimes commis.

En définitive, il est difficile de dire qu'une révolution se soit produite dans la conscience des Serbes, mais l'établissement de la commission et le fait que le

24. Oslobodjenje, 26 août 2006, p. 3. International Criminal Tribunal for the Former Yugoslavia, ICTY Weekly Press Briefing, 30 août 2006, accessible sur http://www.un.org/icty/briefing/2006/PB060830.htm

25. Intervention pendant la conférence «De la vérité à la justice» organisée à Pale, le 25 mars 2005. Voir Patriot, 28 mars 07 2005, édition en ligne :

http://www.patriotmagazin.com/arhiva/0162/index.htm

26. Voir FENA, Oslobodjenje, Nezavisne novine, 30 mars 2005.

27. Ce débat est toujours en cours, notamment à travers des tentatives parlementaires de rédiger une loi pour établir une Commission de vérité (Komisija za istinu) au printemps 2006. Voir http://www.angelfire.com/bc2/kip/ et http://www.daytonproject.ba

28. FENA, 30 mars 2005.

29. Voir SRNA, 9 juin 2005. 
rapport soit fondé sur des sources fournies majoritairement par les autorités de la RS accréditent l'idée, exprimée dans le rapport, que celui-ci manifesterait la maturité du peuple serbe ${ }^{30}$. Le point le plus positif reste l'engagement personnel de la majorité de membres de la commission, leur courage face aux pressions et aux menaces qui a permis que cette commission ne devienne pas une farce comme on aurait pu le craindre. Bien que la plupart des faits fût déjà bien documentée, il importait que la RS crée elle-même une instance aboutissant à ses propres conclusions, tâche d'autant plus délicate que le gouvernement de la RS envoyait un double message aux membres de la commission, les enjoignant de trouver des informations, à condition que celles-ci ne soient pas trop compromettantes. On peut en revanche regretter le fait que le travail de la commission soit le résultat des fortes pressions exercées par le Haut Représentant, dont les interventions publiques ont été considérées comme un nouveau cas de discrimination des Serbes par la communauté internationale. Ces pressions ont affaibli la légitimité des conclusions du rapport aux yeux de l'opinion serbe en RS. Et ce, d'autant que le texte du rapport n'a pas été distribué au public, que les citoyens ordinaires n'ont pas été informés du détail de ses conclusions, et que ses annexes sont restées strictement confidentielles.

En outre, comme le montrent les accusations de génocide contre les Serbes de Sarajevo lancées au printemps 2005, les travaux de la commission de la RS sur Srebrenica ont engendré des réactions très violentes. Plus les crimes à Srebrenica ont été reconnus, plus se sont exacerbées les revendications d'autres groupes qui, s'inquiétant de la focalisation sur le massacre de Srebrenica et du manque d'attention accordée à leurs proches - victimes d'événements antérieurs à 1995 ou situés dans d'autres parties du pays - demandaient à être, eux aussi, reconnus comme victimes. Cette commission peut donc être considérée comme un modèle pour l'avenir, ou au contraire comme l'exemple de ce qu'il ne faut pas faire, non pour des raisons de personnes mais de principe. En effet, si la société souhaite sincèrement surmonter le passé sanglant des conflits récents, il ne faut pas créer des commissions bosniaques, serbes et croates séparées pour chaque région. La méthode consistant à observer les actions d'un seul groupe sur un territoire géographique restreint a suscité des réactions hostiles de la part des autres groupes nationaux, en l'occurrence la réaction des Serbes, ce qui a donné lieu à une regrettable concurrence entre les victimes. Des appels se font entendre maintenant pour la formation de commissions similaires pour Sarajevo, Bratunac, Visegrad, Foca, Rogatica, Vlasenica, Mostar et d'autres régions encore, suite à d'autres décisions prises par la Chambre de droits de l'Homme et la Cour constitutionnelle ${ }^{31}$.

30. Intervention de Milan Bogdanic, président de la commission, pendant la conférence du ICMP "Mécanismes pour la recherche et la documentation de la vérité », organisée le 20 octobre 2004 à Jahorina.

31. La Chambre des droits de l'Homme de Bosnie-Herzégovine a terminé son mandat le 31 décembre 2003. 
Suite aux appels des groupes en RS et à un blocage du Parlement par tous les représentants serbes, le 25 mai 2006 le Conseil des ministres de la BosnieHerzégovine a décidé de former une Commission d'Etat pour la recherche de la vérité sur les souffrances des Serbes, Croates, Bosniaques, Juifs et des autres groupes à Sarajevo pendant la période de 1992 à 1995 32. Avec une très grande couverture médiatique pour et contre la décision, les membres de la commission ont été nommés en juin 2006, avec la responsabilité de publier un rapport final dans les douze mois ${ }^{33}$.

Parallèlement, divers cercles ont débattu l'idée de créer une Commission vérité et réconciliation pour toute la Bosnie-Herzégovine, mais les groupes de victimes n'ont guère participé à ce débat et la possibilité ne leur a pas été accordée de formuler leurs propres propositions. Mais, quelle que soit la forme qu'ils prennent, ces efforts devront être focalisés sur les besoins des victimes, la recherche de la vérité et de la justice et non pas sur des critères de nationalité. C'est en ce sens qu'essaie d'œuvrer l'ICMP.

\section{Le travail de l'ICMP relatif aux victimes de Srebrenica}

A la fin des conflits en ex-Yougoslavie, le sort de 40000 personnes, dont $71 \%$ ayant disparu en Bosnie-Herzégovine, restait inconnu. L'ICMP considère que nombre d'entre elles ont été victimes d'une politique de disparition forcée et que le fait de les retrouver et de les identifier permet aux proches de faire leur deuil et contribue ainsi à un processus plus large de réconciliation.

L'ICMP a réparti ses activités en deux programmes principaux ${ }^{34}:$ les sciences médico-légales et les initiatives de la société civile. D’une part, en raison des difficultés de l'identification "classique » des corps par les vêtements et les caractéristiques physiques de la personne par rapport à des milliers de squelettes retrouvés dans des fosses communes, l'ICMP a développé un important programme d'identification par ADN. Elle a ouvert une installation médico-légale à Tuzla, le Projet d'identification de la Podrinje (PIP), où sont rassemblés des centaines de restes humains de la région de Srebrenica, et le Centre de réassociation (LRC) à Lukavac, qui a la difficile mission de rendre aux familles des dépouilles mortelles aussi complètes que possible. Il s'efforce de réassocier par des méthodes scientifiques des parties de corps dispersées entre plusieurs sites liés au massacre de Srebrenica. En effet, à la fin de l'année 1995 et au début de l'année 1996, les autorités de RS avaient organisé le déplacement des corps afin de dissimuler les preuves des crimes de masse. Dans les fosses secondaires, les corps ont été entremêlés. C'est donc uniquement par le biais d'une identifica-

33. La commission n'a produit aucun résultat concret dans les premiers six mois de son existence.

Voir l'article de presse «La commission de Sarajevo attend la décision du Conseil des ministres : qui veut empêcher les investigations ? ", Oslobodjenje, 14 février 2007, p. 2.

34. Pour plus d'explications sur ces programmes, voir le site de l'ICMP : http://www.ic-mp.org 
tion par $\mathrm{ADN}$ que les anthropologues peuvent établir un lien entre différents ossements et parvenir à reconstituer les corps de manière fiable. Dans le processus d'identification, l'utilisation des tests ADN implique l'obtention et l'analyse d'échantillons d'os des personnes exhumées et d'échantillons sanguins des proches survivants. Sans ces deux éléments, une adéquation entre les formules d'ADN ne peut pas être établie. Pour le seul cas de Srebrenica, l'ICMP a recueilli 20864 échantillons de sang, correspondant à 7789 personnes portées disparues ${ }^{35}$, et 7054 échantillons d'os, transmis par les autorités responsables à la Division pour la coordination de l'identification de Tuzla. 5901 rapports de correspondance entre codes $\mathrm{ADN}$ de sang et d'os ont été établis, pour un total de 3743 individus différents, et 2002 réassociations d'éléments séparés de squelettes ont été réalisées. 2644 cas d'identifications sont clos à ce jour et 2526 corps ont été enterrés, dont 2374 au mémorial de Potocari 36.

D'autre part, dans son programme pour les initiatives de la société civile, l'ICMP travaille avec une centaine d'associations de familles de personnes portées disparues en ex-Yougoslavie. L'objectif de l'ICMP est d'encourager l'engagement et la prise de la parole des familles, notamment pour qu'elles fassent pression sur les autorités et obtiennent la reconnaissance de leurs droits. Depuis 1998, l'ICMP a attribué de nombreuses subventions pour des projets d'associations des familles de disparus de Srebrenica, pour de nombreux ateliers de formation, et d'autres formes de soutien afin qu'elles puissent mieux atteindre leurs buts. Leurs projets sont centrés sur la recherche de la vérité et de la justice, le développement de la Fondation pour le mémorial et le cimetière Srebrenica-Potocari et aussi sur la traduction en justice des criminels de guerre. Pour l'ICMP, il est impératif que les divers groupes de survivants puissent exprimer leurs vues sur le chemin à suivre dans la recherche de la vérité, de la justice et de la réconciliation en ex-Yougoslavie.

35. Selon la base de données de l'ICMP s'appuyant sur les échantillons sanguins, il y a déjà des preuves pour 7789 personnes disparues pendant la chute de Srebrenica en juillet 1995 et le ICMP s'attend à ce que le chiffre final soit environ de 8000 victimes.

36. Chiffres du 25 août 2006 ; voir http://www.ic-mp.org pour des chiffres mis à jour régulièrement. 